\title{
Compact Models and Model Standard for 2.5D and 3D Integration
}

\author{
Qiaosha Zou, Yuan Xie \\ Computer Science and Engineering \\ The Pennsylvania State University \\ \{qszou, yuanxie\}@cse.psu.edu
}

\begin{abstract}
3D integration is an emerging interconnect technology that can enable the continuation of performance scaling and the reduction of form factors. There are various approaches for 3D integration, including system-in-package (SiP), TSVbased 3D ICs, monolithic 3D ICs, and inductance/capacitance coupling 3D ICs, among which TSV-based 3D IC is the most promising one. This paper provides a summary of previous work on electrical modelings for 3D IC, with an emphasis on two key interconnect approaches: TSVs and RDLs. Based on prior work, we describe a compact model standard to facilitate a generic modeling approach for future 3D ICs.
\end{abstract}

\section{INTRODUCTION}

By utilizing the high density through-silicon vias (TSVs), three dimensional integrated circuits (3D ICs) and 2.5D designs are promising solutions to handle problems with continuously transistor scaling according to Moore's law, pushing circuit designs into the "More-than-Moore" regime. In additional to the low latency and high bandwidth vertical interconnects, 3D ICs can also provide the benefits of smaller footprint, improved packaging density, and heterogeneous stacking $[4,18,6]$. On the other hand, $2.5 \mathrm{D}$ circuits that once treated as an intermediate technology before realizing $3 \mathrm{D}$ integrations has now become a practical alternative implementation of true $3 \mathrm{D}$ IC designs. The interposer in $2.5 \mathrm{D}$ designs can provide high density and low latency connections between chips and $\mathrm{I} / \mathrm{O}$ with affordable design cost and complexity overhead [14]. The disruptive Xilinx Virtex-7 is the first commercial 2.5D FPGA design using interposer. There are four metal layers and the trace width of each layer is $2 \mu \mathrm{m}$ [2]. As a comparison, for the connections with PCB (printed circuit board) for DDR4, the trace thickness and width for the signal layer are $0.6 \mathrm{mil}$ (a mil is $1 / 1000$ of an inch) and $6 \mathrm{mil}$, respectively, and the corresponding singleended impedance is about $39 \mathrm{ohms}$.

There are several enabling components in $3 \mathrm{D}$ and $2.5 \mathrm{D}$ ICs, including through-silicon-vias (TSVs), redistribution layers (RDLs), micro-bumps, monolithic 3D ICs, and inductance/capacitance coupling 3D ICs. Among these components, the high aspect-ratio TSVs, providing the connec-

Permission to make digital or hard copies of all or part of this work for personal or classroom use is granted without fee provided that copies are not made or distributed for profit or commercial advantage and that copies bear this notice and the full citation on the first page. Copyrights for components of this work owned by others than ACM must be honored. Abstracting with credit is permitted. To copy otherwise, or republish, to post on servers or to redistribute to lists, requires prior specific permission and/or a fee. Request permissions from Permissions@acm.org.

SLIP '14, June 01 - 02 2014, San Francisco, CA, USA Copyright 2014 ACM 978-1-4503-3053-4/14/06 \$15.00.

http://dx.doi.org/10.1145/2633948.2633955 . tions between active dies, is the key technology for $3 \mathrm{D} / 2.5 \mathrm{D}$ ICs. These newly introduced components influence the circuit performance in a way that traditional 2D electrical models cannot capture. Simple and accurate 3D interconnect models are required for better circuit performance predictions and design exchanges. Plenty of previous work have been done to explore the electrical, mechanical, and thermal characteristic of TSVs, however, these work are based on specific assumptions and the conclusions are limited to the corresponding assumptions (TSV size, connection methods, etc.). In practical, diverse fabrication processes, geometries, and materials have impacts on the characteristics of interconnect components, such as electrical properties, mechanical properties and thermal properties [4, 15]. Moreover, most of the $3 \mathrm{D}$ electrical behavior studies rely on the timeconsuming multi-physics analysis tools. A compact 3D $/ 2.5 \mathrm{D}$ model standard for electrical behavior is urgently needed to aid electronic design automation tools for 3D design modeling and design validation.

In this survey paper, previous work on electrical modelings of 3D designs are reviewed, emphasizing on two key indispensable interconnect components: TSVs and RDLs. The models of TSVs are categorized into two levels according to the model complexity and accuracy. Three types of RDL transmission lines are discussed and one equivalent circuit model is introduced for model simplicity. Modeling and verification methods are explained briefly as well as the given specific assumptions for better understanding.

The following sections are organized as belows. Section 2 introduces previous TSV electrical models in both low frequency and high frequency region. Section 3 illustrates the electrical characteristic of three types of RDL in interposers and briefly introduces the modeling of micro-bumps. In section 4 we describe our proposed 3D compact model based on the previous studies.

\section{REVIEW OF TSV ELECTRICAL MODELINGS}

The geometry of TSVs influences the final modeling accuracy. Most papers treat the TSV as equivalent cylindrical structure [12, 9, 16, 19]. The assumption of abstracting TSV structure into a simple cylindrical structure is examined by Savidis et al. [12]. This study compares the electrical parameters extracted from a cylinder structure contains both top and bottom copper landing to the proposed simple structure without landing by using Ansoft electromagnetic simulation tool. The results show that only less than a $7 \%$ difference in the final RLC (resistance, inductance, and capacitance) value, indicating that using simple cylindrical structure is sufficient for accurate TSV modeling. However, this paper only tested results with frequency as high as $1 \mathrm{GHz}$ under 
certain temperature. The conclusion may not be applicable for higher frequency beyond this point.

For circuit performance (delay, power consumption, heat dissipation) estimation, RLC model is the most straight forward modeling method by viewing the device as an equivalent circuit. The RLC model for single TSV and coupled TSVs with closed-form expressions are given for various analysis and design purposes $[8,5,12,16]$. In these models, the resistance and inductance are in serial and the capacitance exists between the TSV and the substrate. However, these models treat silicon substrate as an ideal conductor (RC model) or dielectric (RLC model) which may bias the electrical value. The skin effect and eddy currents in silicon should be taken into consideration for the TSV modeling at $\mathrm{AC}$ and high frequencies [20]. Skin effect means the current density drops by a certain factor below the surface of a conductor. It has significant impact on the high frequency resistance. Under this circumstance, the RLCG model is usually used for high frequency TSV modeling [20, 11]. This model contains two components: admittance per unit TSV height which consists of conductance and susceptance; impedance per unit TSV height which is composed of resistance and reactance. In this section, both the RLC and RLCG models are introduced. In the RLC modeling, in addition to an isolated TSV, the scenario of coupling between TSVs are also considered.

\subsection{The RLC model for Single TSV}

For a single TSV (without considering the influence from other TSVs), the RLC model is shown in Figure 1(a). Capacitances exist between the TSV and the adjacent substrate while resistance and inductance are in serial along the TSV. Considering the model accuracy and complexity, the resistance modeling is directly derived from the equivalent transmission line. Since the silicon has finite conductivity and the oxide dielectric layer, the modeling of capacitance between TSV and the silicon substrate should consider the MOS (metal-oxide-semiconductor) effect. For the inductance, an empirical close-form equation is given.

The resistance can be described as a function of TSV conductivity $(\sigma)$, TSV length $(l)$, and radius $(r)$ :

$$
R_{t s v}=\frac{l_{v}}{\sigma \pi r_{v}^{2}}
$$

The effective capacitance in TSV is the depletion capacitance $\left(C_{d e p}\right)$ and the oxide capacitance $\left(C_{o x}\right)$ acting in serial.

$$
C_{T S V}=\frac{C_{o x} C_{d e p}}{C_{o x}+C_{d e p}}
$$

The oxide capacitance is related to the permittivity of silicon and oxide and the TSV gemometry, which can be capture as follows:

$$
C_{o x}=\frac{2 \pi \varepsilon_{S i} \varepsilon_{o x} L_{T S V}}{\ln \left(1+\frac{T_{t o x}}{R_{T S V}}\right)}
$$

Nevertheless, the depletion capacitance is infeasible to express in a single close-form equation. The value is determined on the depletion region width, which relies on the flat bend voltage and the bias voltage in silicon substrate. In general, the depletion capacitance is given as follows while

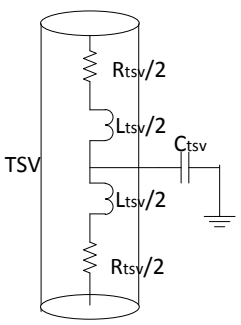

(a) (b)
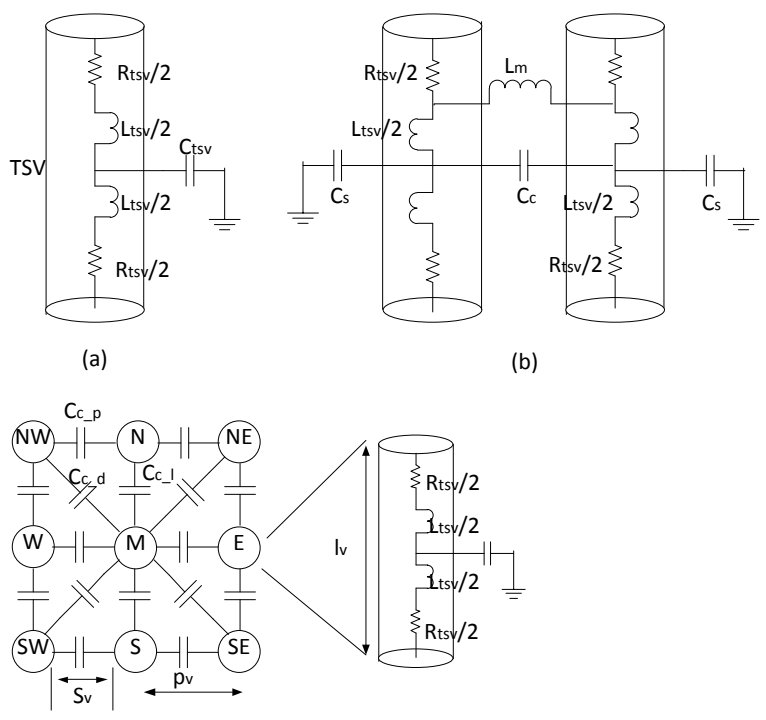

(c)

Figure 1: The resistance, inductance, and capacitance model for (a) A single TSV; (b) Two coupled TSVs; (c) A TSV bundle[16].

the flat bend voltage calculation can refer to the MOS effect.

$$
C_{d e p}=\frac{2 \pi \varepsilon_{S i} L_{T S V}}{\ln \left(1+\frac{T_{t o x}+W_{d e p}}{R_{T S V}}\right) \sqrt{1+\frac{V_{t s v}}{V_{t h}}}}
$$

The final effective capacitance is a function of its geometry and the effective permittivity $\left(\epsilon_{0}\right)$ of surrounding dielectric liner. The following expression is based on empirical value which assumes the thickness of dielectric layer is smaller than $1 \mu m[16]$ :

$$
C_{t s v}=\frac{63.36 \varepsilon_{0} l_{v}}{\ln \left(1+5.26 \frac{l_{v}}{r_{v}}\right)}
$$

When the TSV is treated as a lossy transmission line in the model, the inductance has great impact on signal propagation delay. The propagation delay study by Khalil et al. [9] shows that without the presence of inductance in TSVs, the average error is $55.2 \%$ higher than RLC model with the value of the distributed RLC. The inductance of an isolated TSV is depended on the geometry parameters. It can be expressed as follows [16]:

$$
L_{t s v}=\frac{\mu l_{v}}{2 \pi} \ln \left(1+\frac{2.84}{\pi} \frac{l_{v}}{r_{v}}\right)
$$

All the above empirical closed-form equations predict the RLC values within maximum $6 \%$ error verified by a $3 \mathrm{D} / 2 \mathrm{D}$ quasi-static electromagnetic-field solver tool. By using these closed-form expressions, the resistance, capacitance, and inductance values in a single TSV can be easily calculated for fast circuit simulation.

\subsection{The RLC Model for Coupled TSVs}

The equivalent RLC circuit of two coupled TSVs and a TSV bundle is shown in Figure 1(b) and (c), respectively. For coupled TSVs, the resistance expression is the same as the $\mathrm{R}$ in an isolated single TSV since coupling effect has 
no impact on the resistance. But for the inductance and capacitance, the inter-via coupling plays an important role. Capacitance and inductance are divided into two parts: self parameter and mutual parameter.

The capacitance terms of the whole coupled bundle TSV can be expressed as follows [16]:

$$
C_{\text {bundle }}=\left[\begin{array}{cccc}
C_{1,1} & -C_{1,2} & \ldots & -C_{1, n} \\
-C_{2,1} & C_{2,2} & \ldots & -C_{2, n} \\
\vdots & \vdots & \ddots & \vdots \\
-C_{n, 1} & -C_{n, 2} & \ldots & C_{n, n}
\end{array}\right]
$$

The diagonal elements represent the sum of self and intervia coupling capacitances. This capacitance matrix is sparse because only diagonal elements and elements for nearest neighbors contain meaningful values. From the experiments, in a $7 \times 7$ TSV bundle, coupling effects among nearest neighbors are much significant than those that are non-adjacent.

The self capacitance formula is different from the isolated TSV, which is given as:

$$
\begin{aligned}
C_{s}= & C_{T S V}-k_{1} C_{T S V} e^{\left(k_{2} \frac{p_{v}}{r_{v}}+k_{3} \frac{p_{v}}{l_{v}}\right)} \\
& {\left[k_{4}\left(\frac{L_{v}}{r_{v}}\right)^{k_{5}}+k_{6}\left(\frac{p_{v}}{r_{v}}\right)^{k_{7}}+k_{8}\right] }
\end{aligned}
$$

where the parameters from $k_{1}$ to $k_{8}$ are empirical constants. However, these constants are based on the simulation results and vary with different TSV configurations, making it difficult to be directly applied in circuit simulations. When $k_{2}$ and $k_{3}$ are negative and $p_{v}$ approaches infinity, $C_{s}$ equals to $C_{T S V}$.

The formula for the coupling capacitance where $i \neq j$ in the matrix is given as follows. This model has maximum $6 \%$ error from the simulation results.

$$
\begin{aligned}
& C_{\text {coupled }}=\frac{k_{1} \epsilon_{0} l_{v}}{\ln \left(k_{2} \frac{p v}{r v}\right)} \\
& {\left[1+k_{5}\left(\frac{L_{v}}{r_{v}}\right)^{k_{6}}+k_{3}\left(\frac{p_{v}}{r_{v}}\right)^{k_{4}}+k_{7}\left(\frac{p_{v}}{l_{v}}\right)^{k_{8}}\right]}
\end{aligned}
$$

The coupling inductance can also be defined by the matrix similar to the coupling capacitance. Different from the capacitance, inductive coupling has longer range so the matrix is not sparse. The mutual inductance between any two TSVs can be captured with the following formula:

$$
L_{m}=0.199 \mu l_{v} \ln \left(1+0.438 \frac{d_{v}}{l_{v}}\right)
$$

where $d_{v}$ is the center-to-center distance between two TSVs. The maximum error for coupling inductance is within $8 \%$.

\subsection{The RLCG Model for A Pair of TSVs}

The equivalent distributed circuit model is shown in Figure 2(a), the simplified model is given in Figure 2(b). The impedance, which is represented by $Z$, is similar to the resistance and inductance in serial in RLC model. Capacitance exists inside the depletion region between the TSV and the silicon substrate. Admittance, which is represented by $Y$, exists in the silicon substrate between two adjacent TSVs. In this figure, $Y_{\text {open }}$ is the input admittance between ports 1 and 2 if ports 3 and 4 are open while $Z_{\text {short }}$ represents the impedance between ports 1 and 2 when ports 3 and 4 are short circuited.

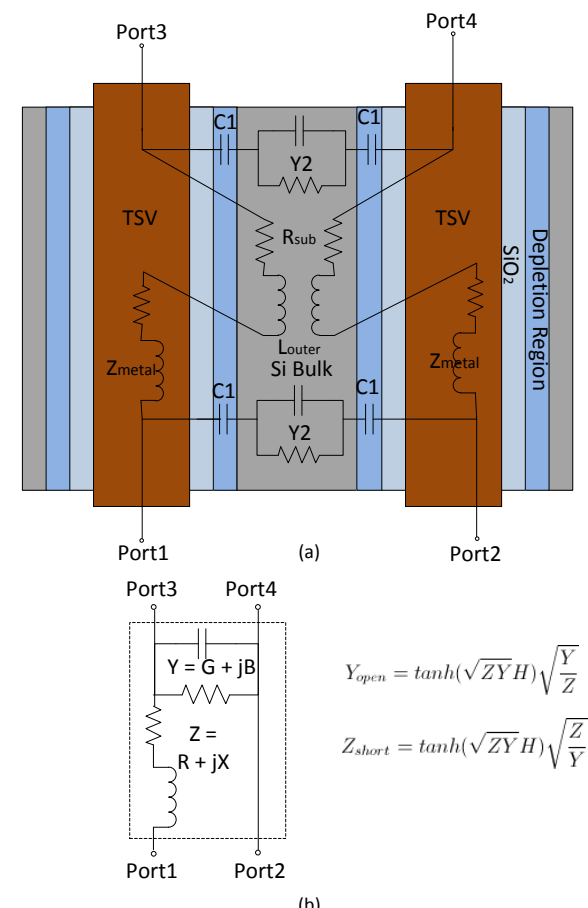

Figure 2: The RLCG model for TSVs: (a) the equivalent distributed RLCG model of two coupled TSVs; (b) a simplified distributed transmission line model [20].

Admittance of TSV in RLCG model. The admittance (CG) per unit TSV height can be treated as two components functioning in serial: one is the silicon depletion region $\left(C_{1}\right)$ and the other is the coupling admittance $\left(Y_{2}\right)$ in the bulk silicon. The admittance formula is shown in the following equation:

$$
Y=\left[2\left(j \omega C_{1}\right)^{-1}+Y_{2}^{-1}\right]^{-1}
$$

where $\omega$ is the radial frequency. Since there are two TSVs contribute to $C_{1}$ in serial with $Y_{2}$, the equation contains a factor of 2 before $C_{1}$.

The capacitance in the depletion region $\left(C_{1}\right)$ can be captured with the following formula:

$C_{1}=\left[\frac{1}{2 \pi \varepsilon_{O X}} \cdot \ln \left(1+\frac{t_{O X}}{r_{v i a}}\right)+\frac{1}{2 \pi \varepsilon_{S i}} \cdot \ln \left(1+\frac{\omega_{\text {dep }}}{r_{v i a}+t_{O X}}\right)\right]^{-1}$

where $\varepsilon_{O X}$ and $\varepsilon_{S i}$ represent the permittivity of dielectric and silicon substrate, respectively. The geometrical parameters $r_{v i a}, t_{O X}$, and $\omega_{d e p}$ are the via radius, isolation layer thickness and silicon depletion width.

The coupling admittance in Si bulk can be illustrated with following equation ( $\sigma_{S i}$ is the conductivity of silicon):

$$
Y_{2}=\pi\left(\sigma_{S i}+j \omega \varepsilon_{S i}\right) / \operatorname{arccosh}\left(\frac{d}{2} /\left(r_{v i a}+t_{O X}+\omega_{d e p}\right)\right)
$$

The CG model is verified with a 2-D quasi-electrostatic simulation tool. The results suggest that at low frequencies, if the depletion region is not considered, the error is not negligible. However, the difference is not so significant at high frequencies. 


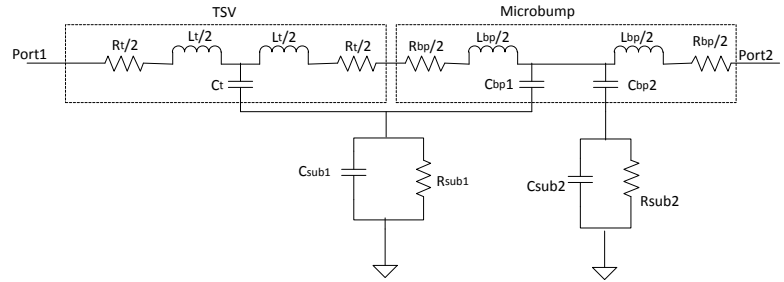

Figure 3: The RLC model of micro-bumps[17].

Impedance of TSV in RLCG model. The serial impedance (RL) per unit height is not so straight forward. The final expression results are shown without detail deduction steps. For simplicity, the serial impedance can be treated as the sum of three components: the inner impedance of $\operatorname{TSV}\left(Z_{\text {metal }}\right)$; the outer inductance $\left(L_{\text {outer }}\right)$; and the resistance due to eddy currents in silicon substrate $\left(R_{\text {sub }}\right)$ :

$$
Z=2 Z_{\text {metal }}+j \omega L_{\text {outer }}+R_{\text {sub }}
$$

The equations for these three components are given as followings:

$$
Z_{\text {metal }}=\frac{(1-j) \cdot J_{0}\left((1-j) r_{\text {via }} / \delta_{\text {metal }}\right)}{\sigma_{\text {metal }} \cdot 2 \pi r_{v i a} \delta_{\text {metal }} \cdot J_{1}\left((1-j) r_{\text {via }} / \delta_{\text {metal }}\right)}
$$

$$
\begin{aligned}
& R_{\text {sub }} \approx \frac{\omega \mu}{2} \\
& \quad \operatorname{Re}\left[H_{0}^{(2)}\left(\frac{1-j}{\delta_{S i}} 9 r_{v i a}+t_{O X}+\omega_{\text {dep }}-H_{0}^{(2)}\left(\frac{(1-j) d}{\delta_{S i}}\right)\right)\right] \\
& L_{\text {outer }} \approx \frac{\mu}{\pi} \ln \left(\frac{r_{v i a}+t_{O X}+\omega_{\text {dep }}}{r_{v i a}}\right)+\frac{\mu}{2} \\
& \quad \operatorname{Im}\left[H_{0}^{(2)}\left(\frac{1-j}{\delta_{S i}}\left(r_{v i a}+t_{O X}+\omega_{\text {dep }}\right)-H_{0}^{(2)}\left(\frac{(1-j) d}{\delta_{S i}}\right)\right]\right.
\end{aligned}
$$

where $\mu$ is the permeability in either TSV or silicon, $J_{0}$ is the 0th order Bessel function of the first type, $H_{0}^{(2)}$ is the 0th order Hankel function of the second type, $\delta_{\text {metal }}$ and $\delta_{S i}$ are the damping parameters for TSV and silicon, respectively.

The results are compared with simulation results, indicating that taking the skin effect into consideration is of great importance for high frequency analysis since the higher frequency resistance is dominant.

As technology scales, the diameter and pitch of TSVs is expected to reduce. However, the substrate thickness almost remains the same as predicted by the International Technology Roadmap for Semiconductors (ITRS). When the radius of TSVs is reduced, C, G, and L do not change much due to the proportional scaling of geometrical parameters. Nevertheless, resistance increases significantly when the frequency grows from $1 \mathrm{GH}$ to $100 \mathrm{GHz}$ due to the reduced TSV cross area.

In terms of circuit performance sensitivity, capacitance has the most important impact on circuit behavior while resistance is of the least importance. The circuit exhibits the short-transmission line behavior on signal propagation, which indicates that simple RLC model is enough for delay and signal rise/fall calculation. However, the L and G are crucial factors for the estimation of voltage variations in $V_{D D}$ and $G N D$. More accurate whole circuit performance evaluation can only be done with all RLCG components.

\subsection{Model Validation Methods}

The proposed analytical models require model validation either from measurement results or well-established simulation results. In general, four validation methods are applied in the previous studies: $2 \mathrm{D} / 3 \mathrm{D}$ electromagnetic solver $[9$, $12,4,16,19,10,13]$; 3D electrostatic simulator [3, 20]; vector network analyzer for frequency domain [5]; and groundsignal-ground (GSG) probing [7, 11].

\section{REVIEW OF RDL AND MICRO BUMP ELECTRICAL MODELING}

Although TSV is the key enabling component in $2.5 \mathrm{D} / 3 \mathrm{D}$ structures, electrical models of other components (microbumps, RDLs, C4, etc.) are necessary for full chip and full package analyses. Several work have been done to model these components $[17,3,10,7,13]$.

Among these components, RDL is the most important one to enable the submicron width and spacing for high density connection in the silicon interposer. The fabrication process of RDL is similar to the back-end-of-line (BEOL) process. There are three different types of transmission lines in RDL, namely microstrip line, stripline, and coplanar waveguide (CPW) line. The major difference rises from the relative position and orientation of signal and ground lines. These three types exhibit diverse insertion loss and return loss [7]. In general, Microstrip line and CPW line have better electrical performance and lower cost. From the performance evaluation, it is found that the dielectric layer thickness is more important than the trace width in terms of the trace impedance.

The metal trace in RDLs can be treated as traditional metal layers for signal transmission with resistance and capacitance. The theoretical values of resistance for RDL can be calculated as follows [10]:

$$
R_{T h}=R_{R D L}+\frac{R_{\text {Ground }}}{2}=\alpha \frac{1}{w_{R D L} * t_{R L D}} \frac{3}{2}
$$

where $w_{R D L}$ and $t_{R D L}$ are the width and the oxide thickness of the metal layers. The resistance value of BEOL can be obtained in the similar way. The capacitance of RDLs has not been deliberately studied in the previous work. However, for simplicity, we can only consider the oxide capacitance due to the dielectric layer.

Micro-bump cannot be simply treated as metal layers due to its unique geometry. In previous study [17], the microbump is modeled as an equivalent RLC circuit similar to the TSV modeling. This model uses the same equations that are used in TSV RC calculation. The micro-bump model contains resistance and inductance in serial along the structure from input to output port as shown in Figure 3. Two capacitances exist between micro-bump and connected tiers. One of the capacitance represents the capacitance between microbump and substrate of the first tier while the other captures the capacitance between micro-bump and the substrate of the second tier. However, this micro-bump model is not accurate because the geometry properties is ignored. Different from metal trace and TSVs, micro-bump is a sphere-like structure which cannot be simply treated as a transmission line or a cylinder. 
Most of the previous work focus on modeling micro-bumps, RDL, and BEOL in a separate fashion, the full chip modeling and analysis with all these components has not been fully explored yet. The resistance path of TSV-RDL-bump and the corresponding insertion loss and return loss are evaluated [13]. The resistance discontinuity has great influence on the impedance and return loss. Therefore, a modified ground-shape is proposed to smooth the impedance profile.

\section{2.5D/3D INTERCONNECT COMPACT MODEL PARAMETERS}

In this section, the extracted parameters are listed for both geometry and electrical modeling of interconnect components. The geometry parameters of interconnect components are one of the dominant factors in the $2.5 \mathrm{D} / 3 \mathrm{D}$ electrical compact modeling. Most of the analytical modelings are based on the extracted geometry parameters. The commonly used extracted geometry parameters of throughsilicon vias, micro-bumps, and transmission lines (RDLs and BEOLs) are given for the reference of further electrical modeling. Most of the electrical parameters are closely depended on the interconnect component materials, such as the electrical conductivity and permittivity. Both the extracted parameters used as model input and the calculated values as model output are given for circuit simulation.

\subsection{Parameters of Through-Silicon Vias}

The key vertical interconnect component in $2.5 \mathrm{D} / 3 \mathrm{D}$ is through-silicon vias (TSVs). The TSV structure contains three parts: metal filling, insulator layer, and silicon substrate. Dielectric layer made from oxide material is surrounding the TSVs metal filling. Between the liner and silicon substrate, depletion region is formed due to the increased bias voltage when the bias voltage is larger than flat bend voltage. The surrounding layers of the filling metal in TSVs have great influence on the electrical behavior. Therefore, during structure extraction, these layers should also be considered and modeled.

In the ideal case, the TSVs shape can be simply extracted as cylindrical structure, which is demonstrated in Figure 4(a). Practically, considering the process variation and manufacturing limitation, real TSV should be modeled as cone structure as shown in Figure 4(b) and the assumption of this structure can be supported by the TSV SEM image from IMEC. In the cone structure, the bottom radius of TSVs is smaller than the upper radius, and the oxide layer has the similar changes (upper radius is larger) but with steeper decrement ratio. These effects are included in the process variation section. The first part of Table 1 demonstrates the TSV geometry parameters under ideal cases.

Besides the dielectric layer thickness, the trapped charges in oxide liner also influences the flat bend voltage and results in different depletion width. Due to the important role of TSVs, the electrical modeling of TSV should be accurate and sophisticated. Since in real designs, the TSV bundle that contains multiple TSVs is used for signal transmission, the coupling effect between TSVs should be carefully handled to precisely capture the circuit behavior. The second part of Table 1 lists all the electrical parameters in the model.

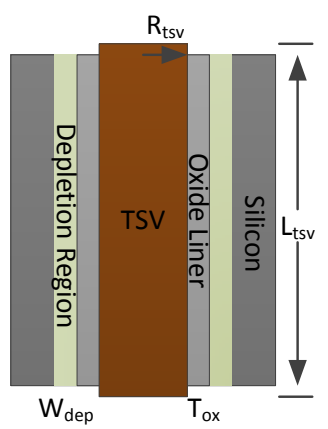

(a) Cylinder TSV

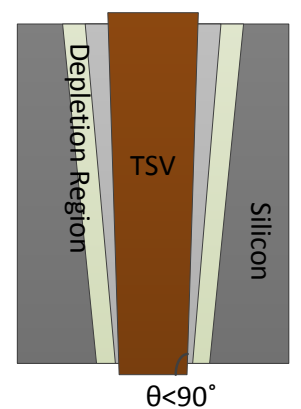

(b) Cone TSV
Figure 4: The typical TSV structure model: (a). TSV is modeled as ideal cylinder; (b). TSV is modeled as cone considering the process limitation and variation.

\subsection{Parameters of RDLs, BEOLs, and Micro-bumps}

Redistribution layers (RDLs) and back-end-of-line (BEOL) can simply be treated as on-chip traditional transmission lines. The RC modeling is more applicable to on-chip interconnect than the package-level connections, such as the metal trace in PCBs. Therefore, the most important parameters include the thickness of the dielectric layer and the length of the metal layer which have impact on the resistance and capacitance as shown in Figure 6.

The extracted geometry parameters for micro-bump is similar to the TSV parameters. Since the micro-bumps are not embedded inside silicon substrate, therefore, the depletion region is not considered. Moreover, large portion of the conductor is copper post and the solder contains only small portion of height (about 1/3). Figure 5 shows the structure of micro-bumps. The geometry parameters of these three components are shown in the first part of Table 2 .

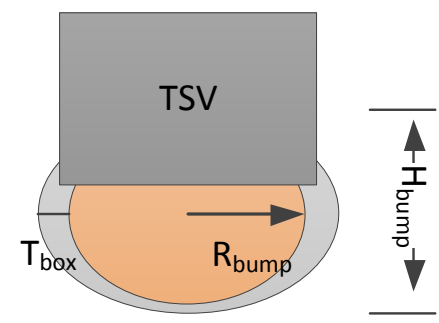

Figure 5: The structure model of micro-bumps.

Compared to TSVs, the electrical modelings of microbumps, RDLs, and BEOLs are relatively simpler and straight forward. Resistance and capacitance are the major factors that influence the signal transmission latency and loss, therefore, in the second part of Table 2, the electrical parameters of each component are listed.

\subsection{Parameters of Process Variation}

In general, the process variation affects the interconnect electrical characteristic through changing the geometry parameters. For example, the electrical modelings of TSV in cylindrical and cone structure are totally different. Moreover, due to the mechanical stress and fabrication process, the micro-bump is more similar to cubic than the ideal sphere. 
Table 1: TSV geometry and electrical parameters list.

\begin{tabular}{|c|c|c|c|}
\hline Category & Parameters & Description & Note \\
\hline \multirow{5}{*}{ Geometry } & $L_{T S V}$ & TSV length & \multirow{2}{*}{ Typical aspect ratio: $1 / 20-1 / 4[1]$} \\
\hline & $R_{T S V}$ & TSV radius & \\
\hline & $T_{t o x}$ & TSV liner layer thickness & \\
\hline & $W_{d e p}$ & TSV silicon depletion width & Depend on bias voltage \\
\hline & $D_{T S V}$ & Distance between two TSVs & Parameter for coupling effects \\
\hline \multirow{6}{*}{ Electrical } & $\sigma_{T S V}$ & TSV conductivity & material related \\
\hline & $\varepsilon_{o x}$ & Oxide liner permittivity & \\
\hline & $\varepsilon_{S i}$ & Silicon permittivity & \\
\hline & $\mu_{0}$ & Free space permeability & default value $4 \pi \cdot 10^{-7} H / m$ \\
\hline & $Q_{\text {trap }}$ & Trapped charges in the oxide liner & charges are between silicon and liner \\
\hline & $V_{t s v}$ & Voltage between TSV and bulk silicon & bias voltage \\
\hline \multirow{9}{*}{ Models } & $R_{t}$ & TSV resistance & \\
\hline & $C_{d e p}$ & TSV depletion capacitance & \\
\hline & $C_{o x}$ & TSV oxide capacitance & \\
\hline & $C_{T S V}$ & TSV capacitance & the serial capacitance of $C_{o x}$ and $C_{d e p}$ \\
\hline & $L_{t}$ & TSV inductance & \\
\hline & $C_{c}$ & Coupling capacitance & \multirow{2}{*}{ coupling effect with more than two TSVs } \\
\hline & $L_{c}$ & Coupling inductance & \\
\hline & $V_{F B}$ & TSV flat bend voltage & \\
\hline & $V_{t h}$ & TSV threshold voltage & \\
\hline
\end{tabular}

Table 2: RDLs, BEOLs, and micro-bumps geometry and electrical parameters list.

\begin{tabular}{|c|c|c|c|}
\hline Category & Parameters & Description & Note \\
\hline \multirow{8}{*}{ Geometry } & $R_{\text {bump }}$ & Micro Bump radius & \multirow{2}{*}{ Micro bump structure extracted as cylinder } \\
\hline & $H_{\text {bump }}$ & Micro Bump height & \\
\hline & $T_{b o x}$ & Oxide liner thickness & \\
\hline & $L_{\text {line }}$ & Length of transmission lines & \multirow{2}{*}{ influence the resistance } \\
\hline & $W_{\text {line }}$ & Width of transmission lines & \\
\hline & $T_{\text {lox }}$ & Oxide thickness of transmission lines & \\
\hline & $L_{\text {type }}$ & The type of transmission line & \multirow{2}{*}{ influence the impedance behavior } \\
\hline & $C_{\text {shape }}$ & Connection shape between line and TSVs & \\
\hline \multirow{3}{*}{ Electrical } & $R_{s}$ & Sheet resistance of transmission lines & \multirow{3}{*}{ material related parameter } \\
\hline & $\varepsilon_{\text {ins }}$ & Liner permittivity for lines and micro bump & \\
\hline & $\sigma_{\text {bump }}$ & Micro bump filling conductivity & \\
\hline \multirow[t]{3}{*}{ Models } & $C_{\text {bump }} / C_{\text {lines }}$ & Component capacitance & $C_{\text {bump }}=\frac{2 \pi \varepsilon_{\text {ins }} H_{\text {bump }}}{\ln \left(1+\frac{T_{\text {box }}}{R_{\text {bump }}}\right)}$ \\
\hline & $R_{b}$ & Micro-bump resistance & $R_{b}=\frac{H_{\text {bump }}}{\sigma_{\text {bump }} \pi R_{\text {bump }}^{2}}$ \\
\hline & $R_{\text {lines }}$ & Transmission line resistance & $R_{\text {lines }}=\frac{R_{s} L_{\text {lines }}}{W_{\text {lines }}}$ \\
\hline
\end{tabular}

Table 3: Process variation related parameters list.

\begin{tabular}{|c|c|c|}
\hline \hline Parameters & Description & Note \\
\hline$R_{T S V}^{m}$ & Minimum TSV radius as cone & \multirow{2}{*}{ Variation in the TSV structure } \\
\hline$T_{b o x}^{m}$ & Minimum oxide liner thickness & \\
\hline$R_{b u m p}^{m}$ & Maximum micro-bump radius under stresses & \multirow{2}{*}{ Variation in the micro-bump structure } \\
\hline$H_{b u m p}^{m}$ & Minimum micro-bump height under stresses & \\
\hline \hline
\end{tabular}




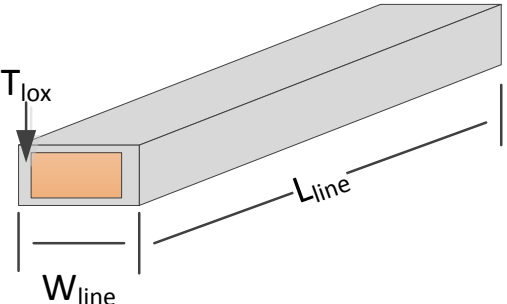

Figure 6: The structure model of transmission lines.

In addition to the geometry variations, electrical parameters are also under influence of the process variation, such as the impurity in silicon substrate. Nevertheless, these impacts can be captured with the previous listed electrical parameters. Due to the various process steps and manufacturing limitations, the major geometry differences are listed in Table 3 .

\section{CONCLUSION AND FUTURE WORK}

Previous work of $2.5 \mathrm{D} / 3 \mathrm{D}$ electrical modeling and validation methods are reviewed and summarized in this paper. The corresponding standard electrical model containing both geometry and electrical parameters is extracted from these previous work. This compact model standard is intended to promote the EDA tool development in 3D IC designs and the design format exchanges between different vendors and designers. Future adoption and integration of this model into existing EDA tools are remained to be explored.

\section{ACKNOWLEDGEMENT}

This work is supported in part by NSF 1218867, 1213052, and 1017277 and SRC grants.

\section{References}

[1] ITRS Report 2011 Edition. http://www.itrs.net/ Links/2011ITRS/Home2011.htm.

[2] Xilinx virtex-7 2.5d fpga. http://www.xilinx.com/ products/silicon-devices/fpga/virtex-7/.

[3] S. Alam, R. Jones, S. Rauf, and R. Chatterjee. Interstrata connection characteristics and signal transmission in three-dimensional (3D) integration technology. In International Symposium on Quality Electronic Design, 2007.

[4] T. Bandyopadhyay, R. Chatterjee, D. Chung, M. Swaminathan, and R. Tummala. Electrical modeling of through silicon and package vias. In IEEE International Conference on $3 D$ System Integration, 2009.

[5] J. Cho, E. Song, K. Yoon, J. S. Pak, J. Kim, W. Lee, T. Song, K. Kim, J. Lee, H. Lee, K. Park, S. Yang, M. Suh, K. Byun, and J. Kim. Modeling and analysis of through-silicon via (TSV) noise coupling and suppression using a guard ring. IEEE Transactions on Components, Packaging and Manufacturing Technology, 1:220-233, 2011.

[6] W. R. Davis, J. Wilson, S. Mick, J. Xu, H. Hua, C. Mineo, A. M. Sule, M. Steer, and P. Franzon. Demystifying 3D ICs: the pros and cons of going vertical. IEEE Test of Comoputer, 22, 2005.

[7] M. Ji, M. Li, J. Cline, D. Secker, K. Cai, J. Lau, P.J. Tzeng, C.-J. Zhan, and C.-K. Lee. 3D Si interposer design and electrical performance study. In DesignCon, 2013.

[8] G. Katti, M. Stucchi, K. De Meyer, and W. Dehaene. Electrical modeling and characterization of through silicon via for three-dimensional ICs. IEEE Transactions on Electron Devices, 57:256-262, 2010.

[9] D. Khalil, Y. Ismail, M. Khellah, T. Karnik, and V. De. Analytical model for the propagation delay of through silicon vias. In International Symposium on Quality Electronic Design, 2008.

[10] J. Roullard, S. Capraro, A. Farcy, T. Lacrevaz, C. Bermond, P. Leduc, J. Charbonnier, C. Ferrandon, C. Fuchs, and B. Flechet. Electrical characterization and impact on signal integrity of new basic interconnection elements inside 3D integrated circuits. In IEEE Electronic Components and Technology Conference, 2011.

[11] C. Ryu, D. Chung, J. Lee, K. Lee, T. Oh, and J. Kim. High frequency electrical circuit model of chip-to-chip vertical via interconnection for 3 -D chip stacking package. In IEEE Topical Meeting on Electrical Performance of Electronic Packaging, 2005.

[12] I. Savidis and E. Friedman. Closed-form expressions of $3-\mathrm{D}$ via resistance, inductance, and capacitance. IEEE Transactions on Electron Devices, 56:1873-1881, 2009.

[13] T. Sung, K. Chiang, D. Lee, and M. Ma. Electrical analyses of TSV-RDL-bump of interposers for high-speed 3D IC integration. In IEEE Electronic Components and Technology Conference, 2012.

[14] M. Sunohara, T. Tokunaga, T. Kurihara, and M. Higashi. Silicon interposer with TSVs (through silicon vias) and fine multilayer wiring. In IEEE Electronic Components and Technology Conference, 2008.

[15] G. Van der Plas, S. Thijs, D. Linten, G. Katti, P. Limaye, A. Mercha, M. Stucchi, H. Oprins, B. Vandevelde, N. Minas, M. Cupac, M. Dehan, M. Nelis, R. Agarwal, W. Dehaene, Y. Travaly, E. Beyne, and P. Marchal. Verifying electrical/thermal/thermomechanical behavior of a 3D stack - challenges and solutions. IEEE Custom Integrated Circuits Conference, 2010.

[16] R. Weerasekera, M. Grange, D. Pamunuwa, H. Tenhunen, and L.-R. Zheng. Compact modelling of through-silicon vias (TSVs) in three-dimensional (3-D) integrated circuits. In IEEE International Conference on 3D System Integration, 2009.

[17] X. Wu, W. Zhao, M. Nakamoto, C. Nimmagadda, D. Lisk, S. Gu, R. Radojcic, M. Nowak, and Y. Xie. Electrical characterization for intertier connections and timing analysis for 3-D ICs. IEEE Transactions on Very Large Scale Integration Systems, 20:186-191, 2012.

[18] Y. Xie, G. H. Loh, B. Black, and K. Bernstein. Design space exploration for $3 \mathrm{D}$ architectures. Journal of Emerging Computer Systems, 2, 2006.

[19] C. Xu, H. Li, R. Suaya, and K. Banerjee. Compact AC modeling and analysis of $\mathrm{Cu}, \mathrm{W}$, and CNT based through-silicon vias (TSVs) in 3-D ICs. In IEEE International Electron Devices Meeting, 2009.

[20] C. Xu, H. Li, R. Suaya, and K. Banerjee. Compact AC modeling and performance analysis of through-silicon vias in 3-D ICs. IEEE Transactions on Electron Devices, 57, 2010. 